Originally published as:

Wadl, M., Heckenbach, K., Noll, I., Ziesing, S., Pfister, W., Beer, J., Schubert, S., Eckmanns, T. Increasing occurrence of multidrug-resistance in acinetobacter baumannii isolates from four german university hospitals, 2002-2006

(2010) Infection, 38 (1), pp. 47-51.

DOI: $10.1007 / s 15010-009-9225-x$

This is an author manuscript.

The definitive version is available at: http://www.springerlink.com 


\title{
Increasing Occurrence of Multidrug-Resistance in Acinetobacter baumannii Isolates From Four German University Hospitals, 2002-2006
}

\author{
M. Wadl, K. Heckenbach, I. Noll, S. Ziesing, W. Pfister, J. Beer, S. Schubert, T. Eckmanns
}

M. Wadl (corresponding author): Postgraduate Training for Applied Epidemiology (PAE, German Field Epidemiology Training Programme), Dept. for Infectious Disease Epidemiology, Robert Koch-Institute, DGZ-Ring 1, 13086 Berlin, Germany; Phone: (+49/30) 18754-3426, Fax: -3533, e-mail: wadlm@rki.de K. Heckenbach, I. Noll, T. Eckmanns: Dept. for Infectious Disease Epidemiology, Robert KochInstitute, Berlin, Germany

S. Ziesing: Institute for Medical Microbiology and Hospital Epidemiology, Hannover Medical School, Hannover, Germany

W. Pfister: Institute of Medical Microbiology, Friedrich-Schiller-University, Jena, Germany

J. Beer: Institute for Medical Microbiology and Epidemiology of Infectious Diseases, University of Leipzig, Leipzig, Germany

S. Schubert: Institute for Infection Medicine, University Medical Center Schleswig-Holstein Campus Kiel, Kiel, Germany

\begin{abstract}
Background: Acinetobacter baumannii can cause severe infections, mainly in critically ill inpatients. Treatment is complicated by multidrug-resistance (MDR). In Germany, to date, little is known on the extent of MDR in A. baumannii isolated from inpatients in German hospitals and potential factors influencing the emergence of MDR.

Materials and Methods: We retrospectively analysed the data of A. baumannii isolates from the inpatients of four German university hospitals, tested for antimicrobial resistance with the broth dilution method between 2002 and 2006. We defined MDR as resistance to three or more classes of recommended drugs. After calculating the proportions of MDR in A. baumannii isolates, we investigated the association between MDR in A. baumannii and year of pathogen isolation, hospital, ward type, specimen and demographics. We performed descriptive analysis and multivariable logistic regression. Additionally, proportions of in vitro drug effectiveness against multidrug-resistant and nonmultidrugresistant $A$. baumannii isolates were determined.

Results: MDR was found in 66 of 1,190 (5.6\%) A. baumannii isolates and increased from 2.1\% in 2002 to 7.9\% in 2006. The highest proportions of MDR were found in hospital A (8.9\%), in intensive care units (7.3\%), in isolates from blood (7.6\%) and in male patients aged 60 years or older (6.6\%). In multivariable analysis, the chance of MDR in $A$. baumannii isolates increased with the successive years of pathogen isolation (odds ratio [OR] 1.3, 95\% confidence interval [Cl] 1.1-1.5) and there was a higher risk of MDR in A. baumannii in intensive care units (OR 1.8, 95\% CI 1.1-2.9). The lowest in vitro antibiotic resistance was found in meropenem, imipenem and ampicillin/sulbactam, with 33, 37 and 39\% for multidrugresistant and 0.4, 1 and 3\% in non-multidrug-resistant $A$. baumannii isolates, respectively.

Conclusions: The increase of MDR in A. baumannii isolates from 2002 to 2006 in four hospitals suggests that clinicians in Germany may expect a rising proportion of MDR in A. baumannii isolates among inpatients. The antimicrobial susceptibility testing of $A$. baumannii isolates against recommended drugs, combined with in-house antimicrobial resistance surveillance, is needed to ensure appropriate treatment.
\end{abstract}




\section{Introduction}

Acinetobacter baumannii is a gram-negative organism and is considered as an emerging hospitalacquired pathogen $[1,2]$. Skin, pharyngeal or faecal colonisation can occur in $1-3 \%$ of healthy humans and potentially creates a patientreservoir as a source for A. baumannii infections in hospital environments. Infections are typically found in critically ill patients. Clinical manifestations most frequently include pneumonia and bloodstream infections. Attributable deaths of patients infected with A. baumannii range from 8 to $23 \%$ and 10 to $43 \%$ in hospitals and intensive care units (ICUs), respectively [3].

The increasing incidence of $A$. baumannii infections during the past several decades can be partially attributed to a rising proportion of susceptible persons as a result of advanced medical support of critically ill and frail patients [1,4]. Survival on dry inanimate surfaces can be between 3 days and 4 months, depending on the strain, and contributes to the successful persistence of Acinetobacter infections in health care settings [5, 6]. A. baumannii has been isolated from hospital equipment, bedding, furniture and staff [7]. Rising rates of antimicrobial drug resistance in A. baumannii isolates are a matter of concern. Resistance to carbapenems - long considered as the first-choice antibiotics in severe A. baumannii infections - exceeded $25 \%$ in both Italy and Turkey, and was higher than $20 \%$ in the UK between 1997 and 2000 [7, 8]. Moreover, the emergence of multidrug-resistance (MDR) complicates treatment.

Antimicrobial resistance varies across countries, as well as on the level of individual hospitals [7]. A US study collecting A. baumannii isolates from 76 centres between January 2004 and September 2005 reported MDR in A. baumannii at 29\% [9]. Large-scale surveillance data on MDR in A. baumannii isolates from Europe is scarce and varying definitions of MDR hamper comparability. Particularly in Germany, to our knowledge, no analyses on large-scale surveillance data of MDR in A. baumannii isolates have been published so far. The German Network

for Antimicrobial Resistance Surveillance (GENARS) continuously collects antimicrobial resistance data from university hospitals using standardised test methods. Our objectives were to determine the proportions of and to identify potential risk factors for MDR in A. baumannii isolates. Additionally, we wanted to assess the in vitro antimicrobial effectiveness of the tested antibiotics against multidrugresistant and non-multidrug-resistant $A$. baumannii isolates to illustrate therapeutic options.

\section{Materials and Methods}

\section{Inclusion Criteria for Data Analysis}

Surveillance data on the antimicrobial susceptibility of A. baumannii isolates sampled between 2002 and 2006 were retrospectively analysed. The data originated from those six German university hospitals which voluntarily participated in the GENARS-project and provided susceptibility data on A. baumannii isolates. They were located in six of 16 German federal states (Baden-Wuerttemberg, Hesse, Lower Saxony, Saxony, Schleswig-Holstein and Thuringia). Exclusively, data on A. baumannii isolates were available; the total number of investigated clinical specimens was not accessible. We only included data from four of the six university hospitals, since those centres tested A. baumannii isolates continuously in the stated time period. The study hospitals had a capacity of 1,117 to 1,411 beds. Only the first collected A. baumannii isolates of inpatients were taken into consideration. Information on the duration between hospital admission and the first sampling for A. baumannii isolation was not available.

\section{Antimicrobial Susceptibility Testing and Definition of MDR}

In each university hospital, antimicrobial susceptibility testing was determined using broth dilution. Therefore, the Micronaut ${ }^{\circledR}$ (Merlin Diagnostika, Bornheim-Hersel, Germany), the Vitek $2 \circledast$ (bioMérieux, Nürtingen, Germany) or an in-house system, following the guidelines from the German Institute for Standardization (DIN) 58940-8, was accepted. DIN breakpoints of susceptibility were used [10]. Internal quality control included the regular use of respective reference strains from the American Type Culture Collection (ATCC). External quality control involved participation in ring tests. We defined MDR as resistance to three or more classes of recommended drugs [2, 11]. If two antibiotics from one class were tested, both needed to be resistant in order to meet the definition. The following antibiotic classes were analysed: beta-lactams (ampicillin/sulbactam, piperacillin/tazobactam), carbapenems (meropenem, imipenem), fluoroquinolones (ciprofloxacin, levofloxacin), cephalosporins (ceftazidime, 
cefepime), aminoglycosides (tobramycin, gentamicin) and the trimethoprim/sulphamethoxazole combination cotrimoxazole.

\section{Data Analysis}

We calculated proportions of MDR in A. baumannii isolates by year of pathogen isolation (2002-2006), hospital (A, B, C, D), ward type (ICU, surgical ward, medical ward, ward with immunocompromised patients), specimen type (respiratory, blood, urine, non-specified, other) and demographics (age, sex) and used the chi-square test to examine possible associations between outcome and explanatory variables. If an explanatory variable consisted of more than two categories, the $p$-value signified the likelihood of differences within the whole group. We considered a p-value $<0.05$ as statistically significant.

For further analysis of potential risk factors for MDR in A. baumannii isolates, we used a multivariable logistic regression model. We chose a stepwise backward regression with a p-value of 0.2 using StataTM version 10

We determined the proportions of multidrug-resistant and non-multidrug-resistant $A$. baumannii isolates in vitro resistant to the tested antibiotics; statistical analysis was performed by using the chisquare test.

\section{Results}

A. baumannii isolates were recovered from 1,190 inpatients. The median age was 52 years and $44 \%$ were female. On average, the number of isolates tested for antimicrobial susceptibility was 238 per year, 298 per hospital, 298 per ward type and 238 per specimen type (Table 1).

MDR was found in 66 of $1,190(5.6 \%, 95 \%$ confidence interval $[\mathrm{Cl}] 4.2-6.8 \%)$ analysed A. baumannii isolates and increased from $2.1 \%(95 \% \mathrm{Cl} 0.05-4.1 \%)$ in 2002 to $7.9 \%(95 \% \mathrm{Cl} 4.5-11.3 \%)$ in 2006. Proportions of MDR in A. baumannii isolates did not rise in all hospitals alike. MDR in A. baumannii isolates increased five-fold between 2002 and 2006 in hospitals A and B and two-fold in hospital D. In one of the hospitals, $11.5 \%(95 \% \mathrm{Cl} 0.0-24.7 \%)$ of $\mathrm{A}$. baumannii isolates were multidrug-resistant in 2003 and no multidrug-resistant isolates were found in the other years studied (Figure 1).

The highest proportions of MDR were found in hospital A (8.9\%, 95\% Cl 5.9-12.0\%), in intensive care units $(7.3 \%, 95 \% \mathrm{Cl} 5.0-9.7 \%)$, in isolates from blood $(7.6 \%, 95 \% \mathrm{Cl} 3.0-12.1 \%)$ and in male patients aged 60 years or older $(6.6 \%, 95 \% \mathrm{Cl} 3.9-9.3 \%)$. Only the differences in the proportions of MDR in A. baumannii isolates by university hospital $(p<0.01)$ (Table 2$)$ reached statistical significance.

Four $(0.3 \%, 95 \% \mathrm{Cl} 0.0-0.7 \%) \mathrm{A}$. baumannii isolates were resistant to all of the tested drugs. They exclusively originated from patients of ICUs in 2004 and 2006. Two isolates came from the same ICU in hospital B and were tested within 1 month of each other.

In multivariable analysis, we identified an increasing risk of MDR in A. baumannii isolates over time of pathogen isolation (odds ratio [OR] 1.3, 95\% Cl 1.1-1.5) and a higher risk of MDR in A. baumannii isolates in ICUs (OR 1.8, 95\% Cl 1.1-2.9). The chance of finding MDR in A. baumannii isolates was lower in hospital $\mathrm{D}(\mathrm{OR} 0.5,95 \% \mathrm{Cl} 0.3-0.9)$ (Table 3). Hospital $\mathrm{D}$ did not differ from the other hospitals regarding year of pathogen isolation, age of patients, ward types or specimen taken.

Antimicrobial Resistance of Multidrug-Resistant and Non-Multidrug-Resistant A. baumannii Isolates The proportions of resistance among multidrug-resistant and non-multidrug-resistant $\mathrm{A}$. baumannii isolates differed for all of the respectively tested drugs $(p<0.001)$. A. baumannii isolates showed the lowest antibiotic resistance against meropenem, imipenem and ampicillin/sulbactam in multidrugresistant isolates (33, 37 and 39\%, respectively) and non-multidrug-resistant isolates $(0.4,1$ and 3\%, respectively) (Figure 2). 


\section{Discussion}

We found higher MDR proportions in A. baumannii isolates from inpatients in ICUs. The proportions of MDR in A. baumannii isolates increased from 2002 to 2006. In hospital D, the chances were lower for inpatients to have a multidrug-resistant $A$. baumannii isolate. We did not find any associations between MDR in A. baumannii isolates and specimen types or demographics.

Higher proportions of MDR in A. baumannii isolates among patients in ICUs were also found in other studies and can be explained by a more frequent use of invasive procedures like catheterisation or mechanical ventilation,

a more common use of antibiotics and a higher proportion of critically ill patients compared to other ward types [12].

Our results indicate that clinicians in Germany may expect a rising proportion of MDR in A. baumannii isolates among inpatients. The acquisition of resistance, exchange of genetic elements coding for resistances and the ability of $A$. baumannii isolates to persist and multiply in hospital environments may attribute to this time trend.

After a revision of possible differences between the four hospitals, a specific reason for a lower chance of MDR in hospital D could not be elucidated. Results from a descriptive analysis showed a lower proportion of multidrug-resistant A. baumannii isolates in hospital C compared to hospital D. In multivariable analysis, however, A. baumannii isolates detected in patients from hospital D had the lowest chance of being multidrug-resistant. The reason for this might be the sample size, which was 103 isolates in hospital C and 475 isolates in hospital D.

The results from our data suggest that appropriate antimicrobial therapy of multidrug-resistant $A$. baumannii isolates could be achieved by the use of meropenem, imipenem or ampicillin/sulbactam. However, evidence from in vitro testing does not always result in clinical efficacy.

Limitations of our study include the low number of hospitals from which the data were retrieved. Although the hospitals are located in four different federal states, they are not representative of the 34 existing university hospitals in Germany.

Since we lack clinical information, no differentiation of colonised and infected inpatients was possible. As the genotyping of $A$. baumannii isolates was not performed, and antibiotyping is not as discriminatory [13], possibly, existing outbreaks could not be determined.

Our analyses show the proportions of MDR in a large number of tested A. baumannii isolates from four German university hospitals and the proportions of antimicrobials in vitro still effective. Although the proportion of multidrug-resistant $A$. baumannii isolates we found was rather low compared to the results from the study performed in the US by Sunenshine et al. in 2007 [12], the increase of MDR over time is an alarm signal to be taken seriously. The potential of $A$. baumannii isolates to colonise humans and to persist in hospital environments for many weeks can lead to outbreaks in hospitalised patients. MDR limits therapeutic success.

Every case of multidrug-resistant $A$. baumannii colonisation or infection should result in timely infection- control measures to prevent perpetuation and human-tohuman transmission [7]. The identification of a common source should be prompted and include targeted screening of potentially colonised or infected contact persons, such as health care workers, and sampling of the hospital environment, particularly in ICUs [13]. Considerations in the control and treatment of nosocomial infections due to multidrug-resistant A. baumannii isolates were published by Urban et al. [14], such as the daily review of antibiotic susceptibility of all clinical isolates.

According to $\$ 23$ of the German Protection Against Infection Act [15], the heads of hospitals and institutions for outpatient surgery are obliged to continuously record and evaluate nosocomial infections and the appearance of pathogens with special resistances and multiple resistances in a separate document. These records shall be kept for 10 years and the competent health office shall be given permission to inspect the records on request.

We recommend that hospitals set up an in-houseantimicrobial resistance surveillance system, including the assessment of relevant clinical parameters and to perform routine antimicrobial 
susceptibility testing of $A$. baumannii isolates to guide the selection of empirical antibiotic therapy at the local level and to control the spread of MDR in A. baumannii isolates. Hospitals need to be a safe place for critically ill patients.

\section{Acknowledgements}

During the study period, Maria Wadl was a fellow of the Postgraduate Training for Applied Epidemiology (PAE, German Field Epidemiology Training Programme [FETP]) and the European Programme for Intervention Epidemiology Training (EPIET). We highly appreciate the support and intellectual input of all programme coordinators and especially of Doris Radun on this manuscript. We thank Manuel Dehnert and Matthias an der Heiden (Robert Koch-Institute, Germany) for the statistical analysis support.

\section{References}

1. Bergogne-Bérézin E, Towner $\mathrm{KJ}$ : Acinetobacter spp. as nosocomial pathogens: microbiological, clinical, and epidemiological features. Clin Microbiol Rev 1996; 9: 148-165.

2. Munoz-Price LS, Weinstein RA: Acinetobacter infection. N Engl J Med 2008; 358: 1271-1281.

3. Falagas ME, Bliziotis IA, Siempos II: Attributable mortality of Acinetobacter baumannii infections in critically ill patients: a systematic review of matched cohort and case-control studies. Crit Care 2006; 10: R48.

4. Paul M, Weinberger M, Siegman-Igra $Y$, Lazarovitch T, Ostfeld I, Boldur I, Samra Z, Shula H, Carmeli Y, Rubinovitch B, Pitlik S: Acinetobacter baumannii: emergence and spread in Israeli hospitals 1997-2002. J Hosp Infect 2005; 60: 256-260.

5. Kramer A, Schwebke I, Kampf G: How long do nosocomial pathogens persist on inanimate surfaces? A systematic review. BMC Infect Dis 2006; 6: 130.

6. Wendt C, Dietze B, Dietz E, Rüden H: Survival of Acinetobacter baumannii on dry surfaces. J Clin Microbiol 1997; 35: 1394-1397.

7. Dijkshoorn L, Nemec A, Seifert $\mathrm{H}$ : An increasing threat in hospitals: multidrug-resistant Acinetobacter baumannii. Nat Rev Microbiol 2007; 5: 939-951.

8. Turner PJ, Greenhalgh JM: The activity of meropenem and comparators against Acinetobacter strains isolated from European hospitals, 1997-2000. Clin Microbiol Infect 2003; 9: 563-567.

9. Halstead DC, Abid J, Dowzicky MJ: Antimicrobial susceptibility among Acinetobacter calcoaceticusbaumannii complex and Enterobacteriaceae collected as part of the Tigecycline Evaluation and Surveillance Trial. J Infect 2007; 55: 49-57.

10. Anonymous: Deutsches Institut für Normung e. V. Medizinische Mikrobiologie: Empfindlichkeitsprüfung von mikrobiellen Krankheitserregern gegen Chemotherapeutika - teil 4: Bewertungsstufen für die minimale Hemmkonzentration - Mhkgrenzwerte von antibakteriellen Wirkstoffen. Berlin: Beuth Verlag 2004; DIN 58940-4, Bbl 1.

11. Peleg AY, Seifert H, Paterson DL: Acinetobacter baumannii: emergence of a successful pathogen. Clin Microbiol Rev 2008; 21: 538-582.

12. Sunenshine RH, Wright MO, Maragakis LL, Harris AD, Song $X$, Hebden J, Cosgrove SE, Anderson A, Carnell J, Jernigan DB, Kleinbaum DG, Perl TM, Standiford HC, Srinivasan A: Multidrugresistant Acinetobacter infection mortality rate and length of hospitalization. Emerg Infect Dis 2007; 13: 97-103.

13. Karageorgopoulos $\mathrm{DE}$, Falagas $\mathrm{ME}$ : Current control and treatment of multidrug-resistant Acinetobacter baumannii infections. Lancet Infect Dis 2008; 8: 751-762.

14. Urban C, Segal-Maurer S, Rahal JJ: Considerations in control and treatment of nosocomial infections due to multidrug-resistant Acinetobacter baumannii. Clin Infect Dis 2003; 36: 1268-1274. 15. Anonymous: Act on the Reform of the Communicable Diseases Law (Communicable Diseases Law Reform Act). 2000. Available online at:

http://www.rki.de/cln_151/nn_209238/EN/Content/Prevention/Inf_Dis_Surveillance/inf_dis_down,t emplateld=raw,property=publicationFile.pdf/inf_dis_down.pdf. 


\section{Tables and Figures}

Table 1. Numbers (n) and proportions (\%) of Acinetobacter baumannii isolates detected among inpatients in four university hospitals, Germany, 2002-2006.

\begin{tabular}{|c|c|c|c|c|c|c|c|c|c|}
\hline \multirow[t]{2}{*}{ Total } & \multicolumn{2}{|c|}{ Hospital A } & \multicolumn{2}{|c|}{ Hospital B } & \multicolumn{2}{|c|}{ Hospital C } & \multicolumn{2}{|c|}{ Hospital D } & \multirow{2}{*}{$\begin{array}{l}\text { Total } \\
\text { n } \\
1,190\end{array}$} \\
\hline & $\begin{array}{l}\mathbf{n} \\
336\end{array}$ & $\begin{array}{l}\% \\
28.2\end{array}$ & $\begin{array}{l}n \\
276\end{array}$ & $\begin{array}{l}\% \% \\
23.2\end{array}$ & $\begin{array}{l}\mathrm{n} \\
103\end{array}$ & $\begin{array}{l}\% \\
8.7\end{array}$ & $\begin{array}{l}\mathrm{n} \\
475\end{array}$ & $\begin{array}{l}\% \\
39.9\end{array}$ & \\
\hline \multicolumn{10}{|l|}{ Year of pathogen isolation } \\
\hline 2002 & 54 & 28.3 & 67 & 35.1 & 22 & 11.5 & 48 & 25.1 & 191 \\
\hline 2003 & 51 & 17.1 & 86 & 28.9 & 26 & 8.7 & 135 & 45.3 & 298 \\
\hline 2004 & 56 & 23.3 & 29 & 12.1 & 20 & 8.3 & 135 & 56.3 & 240 \\
\hline 2005 & 84 & 38.2 & 46 & 20.9 & 16 & 7.3 & 74 & 33.6 & 220 \\
\hline 2006 & 91 & 37.8 & 48 & 19.9 & 19 & 7.9 & 83 & 34.4 & 241 \\
\hline \multicolumn{10}{|l|}{ Ward type } \\
\hline Surgical ward & 82 & 24.7 & 77 & 23.2 & 28 & 8.4 & 145 & 43.7 & 332 \\
\hline Medical ward & 88 & 27.4 & 85 & 26.5 & 24 & 7.5 & 124 & 38.6 & 321 \\
\hline Ward with immunocompromised patients & 16 & 27.1 & 17 & 28.8 & 1 & 1.7 & 25 & 42.4 & 59 \\
\hline Intensive care unit & 150 & 31.4 & 97 & 20.3 & 50 & 10.5 & 181 & 37.9 & 478 \\
\hline \multicolumn{10}{|l|}{ Specimen type } \\
\hline Respiratory & 145 & 34.9 & 91 & 21.9 & 41 & 9.9 & 138 & 33.3 & 415 \\
\hline Blood & 34 & 25.8 & 19 & 14.4 & 11 & 8.3 & 68 & 51.5 & 132 \\
\hline Urine & 16 & 13.7 & 43 & 36.8 & 1 & 0.9 & 57 & 48.7 & 117 \\
\hline Other & 140 & 29.5 & 118 & 24.8 & 49 & 10.3 & 168 & 35.4 & 475 \\
\hline Non-specified & 1 & 2.0 & 5 & 9.8 & 1 & 2.0 & 44 & 86.3 & 51 \\
\hline \multicolumn{10}{|l|}{ Demographic information } \\
\hline Age (mean in years) & 47.1 & & 53.6 & & 47.5 & & 54.8 & & 51.7 \\
\hline Female $(\%)$ & 42.0 & & 41.3 & & 48.5 & & 45.3 & & 43.7 \\
\hline
\end{tabular}


Table 2. Numbers ( $n$ ) and proportions (\%) of multidrug-resistance (MDR) in A. baumannii isolates by year of pathogen isolation, hospital, ward type, specimen, age group and sex, Germany, 2002-2006.

\begin{tabular}{|c|c|c|c|c|}
\hline Total & $\begin{array}{l}\text { MDR } \\
\mathrm{n} \\
66\end{array}$ & $\begin{array}{l}\text { MDR } \\
\% \\
5.6\end{array}$ & $\begin{array}{l}\text { Total } \\
\text { n } \\
1,190\end{array}$ & p-value \\
\hline Year of pathogen isolation & & & & 0.11 \\
\hline 2002 & 4 & 2.1 & 191 & \\
\hline 2003 & 16 & 5.4 & 298 & \\
\hline 2004 & 16 & 6.7 & 240 & \\
\hline 2005 & 11 & 5.0 & 220 & \\
\hline 2006 & 19 & 7.9 & 241 & \\
\hline Hospital & & & & $<0.01$ \\
\hline A & 30 & 8.9 & 336 & \\
\hline B & 16 & 5.8 & 276 & \\
\hline D & 17 & 3.6 & 475 & \\
\hline C & 3 & 2.9 & 103 & \\
\hline Ward type & & & & 0.14 \\
\hline Intensive care unit & 35 & 7.3 & 478 & \\
\hline Surgical ward & 17 & 5.1 & 332 & \\
\hline Medical ward & 12 & 3.7 & 321 & \\
\hline $\begin{array}{l}\text { Ward with immuno } \\
\text { compromised patients }\end{array}$ & 2 & 3.4 & 59 & \\
\hline Specimen type & & & & 0.34 \\
\hline Urine & 8 & 6.8 & 117 & \\
\hline Blood & 10 & 7.6 & 132 & \\
\hline Respiratory & 27 & 6.5 & 415 & \\
\hline Non-specified & 2 & 3.9 & 51 & \\
\hline Others & 19 & 4.0 & 475 & \\
\hline Age group & & & & 0.48 \\
\hline $0-59$ years & 30 & 5.1 & 591 & \\
\hline $60+$ & 36 & 6.0 & 599 & \\
\hline Sex & & & & 0.33 \\
\hline Male & 41 & 6.1 & 670 & \\
\hline Female & 25 & 4.8 & 520 & \\
\hline
\end{tabular}

Table 3. Multivariable analysis of factors associated with MDR in A. baumannii isolates in four university hospitals, Germany, 2002-2006.

\begin{tabular}{lll} 
Factor & OR $(95 \% \mathrm{CI})$ & $\mathrm{p}$-value \\
\hline Intensive care units & $1.8(1.1-2.9)$ & 0.03 \\
Year of pathogen isolation & $1.3(1.1-1.5)$ & 0.01 \\
Hospital D & $0.5(0.3-0.9)$ & 0.03
\end{tabular}


Figure 1. Proportions of multidrug-resistance (MDR) in Acinetobacter baumannii isolates by year of isolation and university hospital, Germany, 2002-2006. In total, 66 of 1,190 (5.6\%) A. baumannii isolates were tested as multidrug-resistant.

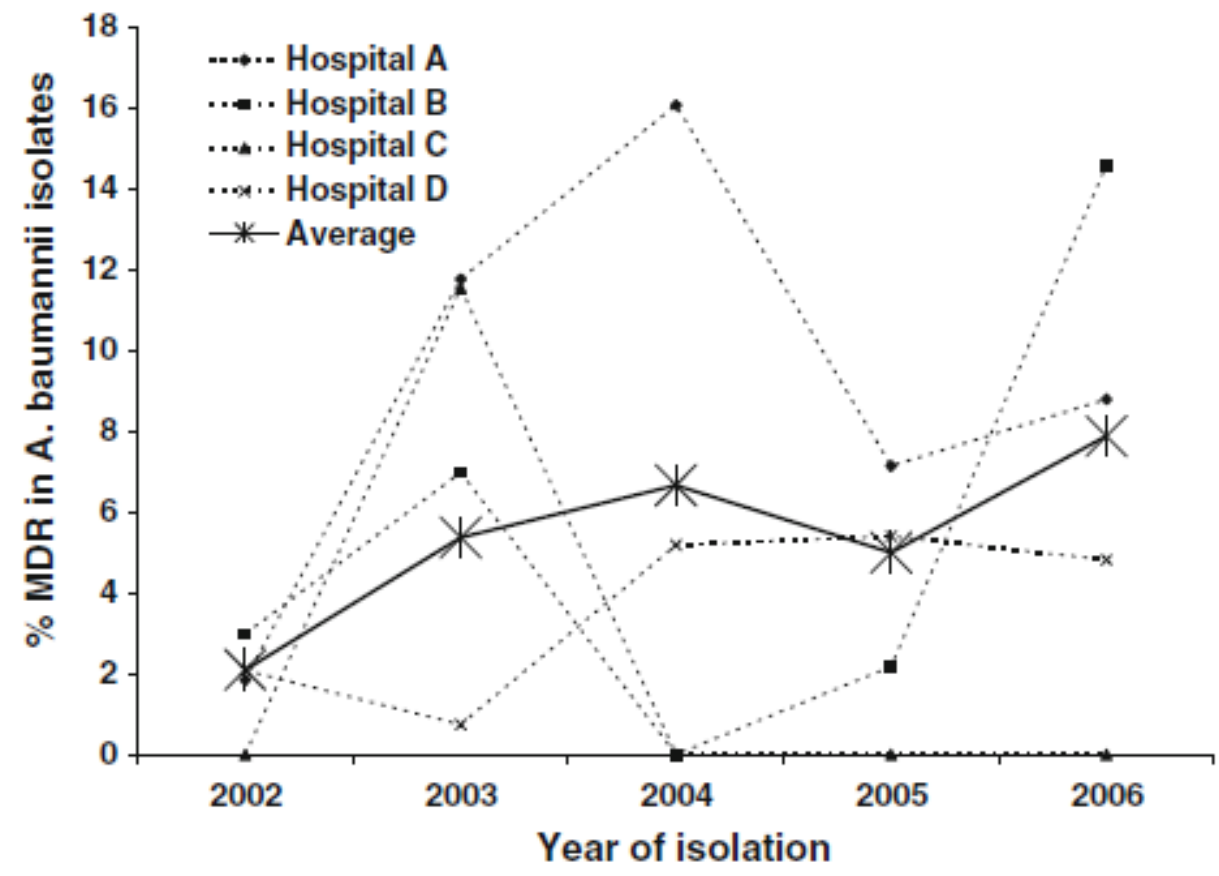

Figure 2. In vitro antimicrobial resistance of multidrug-resistant and non-multidrug-resistant $A$. baumannii isolates in four university hospitals, Germany, 2002-2006.

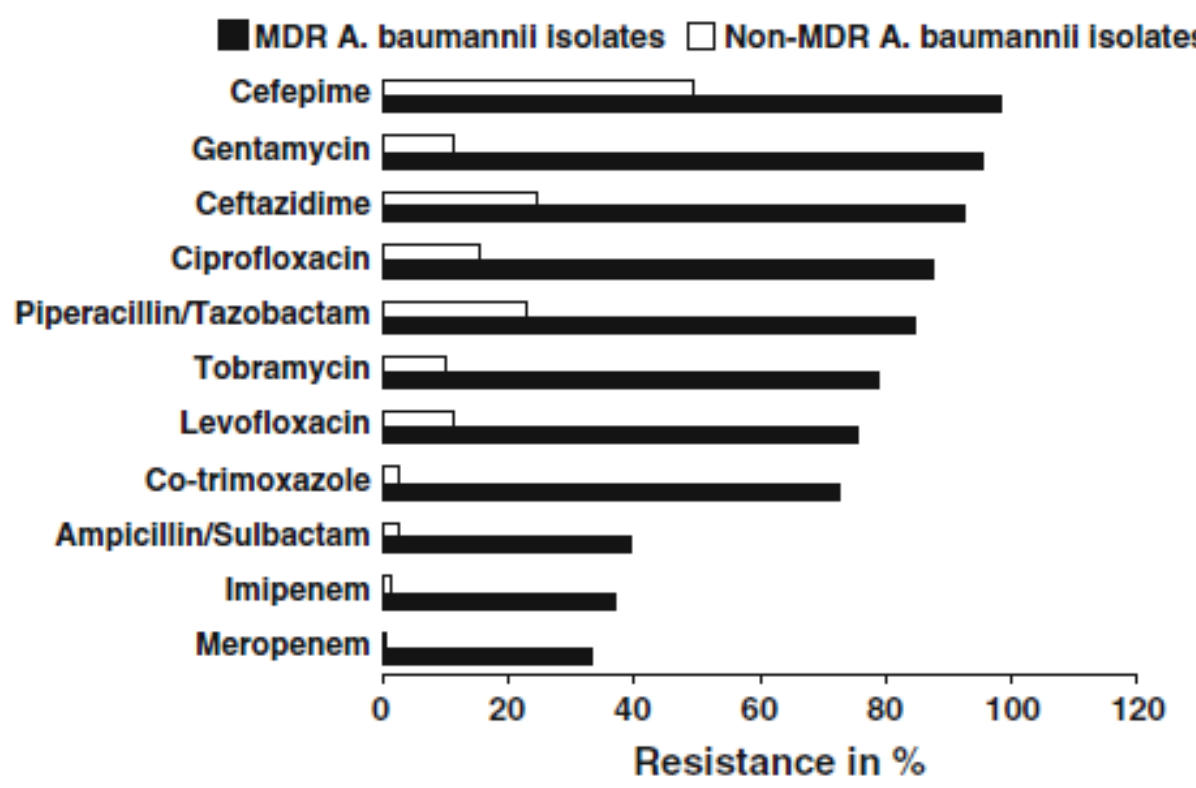

